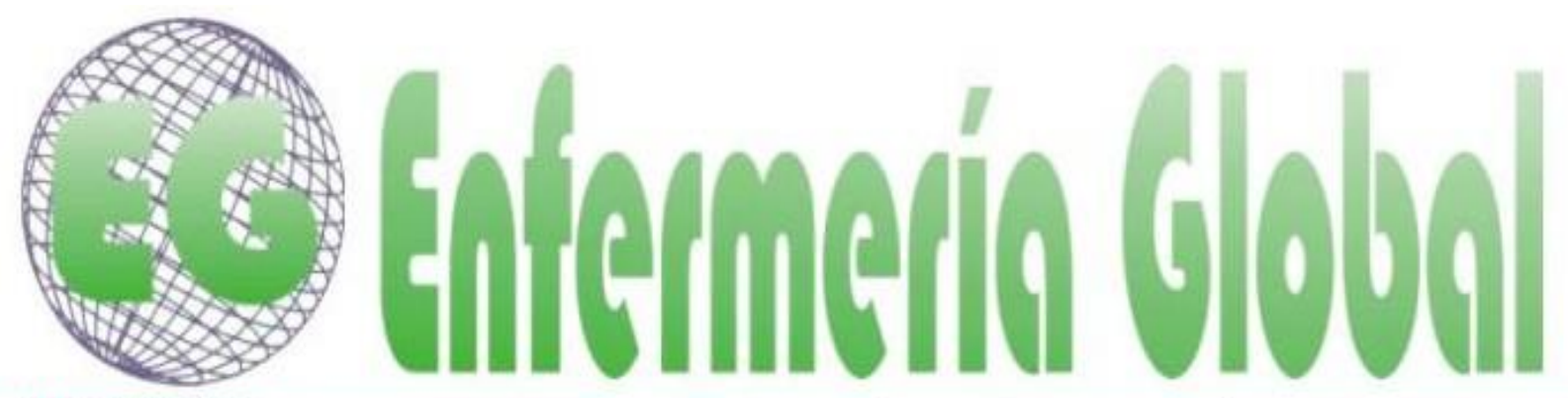

\title{
Tiempo de cicatrización de las heridas crónicas, a propósito de un estudio de prevalencia e incidencia
}

Time of chronic wound healing, as part of a prevalence and incidence study

\section{*Lanau-Roig, Anna **Fabrellas, Núria *Sáez-Rubio, Gema **Wilson, Kate}

*Enfermera. Centro de Salud La Marina. Insitut Catalá de la Salut. E-mail: alanaur.bcn.ics@gencat.cat ${ }^{* *}$ RN. Phd. Profesora Titular Escuela de Enfermería. Universidad de Barcelona.***Enfermera Dip Trans.España.

\section{http://dx.doi.org/10.6018/eglobal.16.2.251311;}

\section{RESUMEN}

Objetivo: Determinar el tiempo medio de cicatrización de las heridas crónicas, así como su prevalencia e incidencia.

Método: Estudio retrospectivo transversal en el ámbito de atención primaria de Barcelona ciudad, con una población de 15.589 habitantes mayores de 15 años. El periodo de estudio fue de 1 de enero a 31 de diciembre de 2013. Se recogió información sobre la etiología de las heridas crónicas y el tiempo necesario para su cicatrización.

Resultados: Fueron localizados 416 registros de lesiones de los cuales el 10,33\% pertenecían a heridas crónicas. La mediana de duración fue de 152 días y la media de 311 días, siendo las úlceras por presión las de mayor duración. Se observó una diferencia estadísticamente significativa $(p<0,05)$ entre la media de duración de las heridas atendidas en el centro de salud y las atendidas a domicilio. La prevalencia fue del 0,28\% y la incidencia del 0,24\%, las lesiones con mayor prevalencia e incidencia fueron las úlceras venosas.

Conclusiones: El tiempo de curación depende del tipo de herida y del lugar de atención. La mayor prevalencia e incidencia se da en úlceras de extremidad inferior.

Palabras clave: úlcera cutánea; cicatrización de herida; atención primaria de salud; atención de enfermería; prevalencia

\section{ABSTRACT}

Aim: To determine the mean healing time of chronic wounds as well as their prevalence and incidence. 
Method: A transversal, retrospective study in the field of primary care in Barcelona city, with a population of 15,589 inhabitants over 15 years of age. The study period was from 1st January to 31 st December 2013. Information on the etiology of chronic wounds and the time needed for healing was collected.

Results: 416 records of injuries were located, of which $10.33 \%$ were chronic wounds. The median duration was 152 days and the mean average was 311 days, with pressure ulcers taking longest to heal. A statistically significant difference $(p<0.05)$ was observed between the mean duration of wounds treated at the health centre and those treated at home. The prevalence was $0.28 \%$ and the incidence $0.24 \%$, the wounds with the highest prevalence and incidence were venous ulcers.

Conclusions: Healing time depends on the type of wound and the point of care. The highest prevalence and incidence occurs in lower-extremity (leg) ulcers.

Keywords: skin ulcer; wound healing; primary health care; nursing care; prevalence.

\section{INTRODUCCIÓN}

Las úlceras cutáneas son una importante causa de morbilidad y mortalidad en todo el mundo, pueden producirse por múltiples causas, entre ellas la diabetes mellitus, la neuropatía periférica, la inmovilidad, la presión, la arteriosclerosis, las infecciones y/o la insuficiencia venosa ${ }^{(1)}$.

Una herida representa la pérdida de continuidad anatómica y fisiológica de la piel. Éstas pueden clasificarse como agudas o crónicas; se considera crónica cuando su curación no se produce en el plazo esperado (2). Según diversas fuentes, herida crónica es aquella cuya curación tiene una duración superior a seis semanas ${ }^{(1,3,4)}$. El 95\% de las úlceras crónicas son de origen venoso, isquémico, neuropático o úlceras por presión (UPP) ${ }^{(2)}$, el resto corresponde a otro tipo de heridas que cicatriza por segunda intención ${ }^{(5)}$.

Las heridas crónicas son un importante problema sanitario que genera graves consecuencias en la calidad de vida de los pacientes, en las personas que los cuidan y en la utilización de recursos, tanto materiales como profesionales. El daño en la piel puede tener un impacto perjudicial en el bienestar general del paciente, a menudo durante largos períodos de tiempo, con algunos pacientes que pasan muchos años en el sistema de atención de salud ${ }^{(6)}$. Los estudios sobre prevalencia e incidencia de heridas crónicas son escasos, pero estos determinan resultados parecidos en los países industrializados ${ }^{(7)}$.

González-Consuegra y Verdú estiman la mediana de antigüedad de las úlceras activas en 210 días, con una enorme variabilidad que oscila desde menos de un año hasta más de 67 años ${ }^{(8)}$.

La prolongación en el tiempo de las heridas crónicas afecta la calidad de vida del paciente y aumenta los gastos asociados a su curación. En Cataluña se ha calculado, que el gasto que realizó el mayor proveedor de servicios de atención primaria, el Institut Catalá de la Salut (ICS), en productos de cura húmeda el año 2010, fue de casi 6 millones de euros ${ }^{(9)}$.

El objetivo de este estudio es conocer el tiempo medio de cicatrización de las heridas crónicas, de los pacientes asignados a un equipo de atención primaria de ámbito urbano. Como objetivo secundario conocer la prevalencia e incidencia de las mismas. 


\section{MATERIAL Y MÉTODO}

Estudio retrospectivo transversal en el ámbito urbano de Barcelona ciudad, desarrollado en la área básica de Salud (ABS) La Marina, con una población asignada mayor de 15 años de 15.589 habitantes. El periodo de estudio fue de 1 de enero a 31 de diciembre de 2013.

La recogida de datos se realizó de julio a octubre de 2014 por un grupo de enfermeras/os de la misma ABS.

La población de estudio fueron las personas con una herida crónica asignada a la ABS la Marina, ya fueran estas atendidas en el domicilio o en el centro de salud.

Se consideraron heridas crónicas, basándonos en la bibliografía revisada ${ }^{(1,3,4)}$, aquellas cuya cicatrización se prolongó más de seis semanas. Se excluyeron las heridas con duración inferior a 42 días y aquellas en que no se pudiera realizar el seguimiento completo por éxitus, ingresos y/o traslados.

Los datos se obtuvieron a través de la estación clínica de atención primaria (eCAP), software utilizado para la gestión de la historia clínica de registro informatizado. La unidad técnica (BASIQ) realizó la explotación directa de las variables códigos diagnósticos CIE-10 (Clasificación Internacional de Enfermedades) y NANDA (NANDA Internacional, Inc) asociados a heridas (tabla I), fecha de alta del diagnóstico, edad y sexo de la persona.

\begin{tabular}{|l|l|}
$\begin{array}{l}\text { Tabla I: Diagnósticos y código } \\
\text { explotación }\end{array}$ & vinculado para su \\
\hline ClE-10 & Código \\
\hline Quemadura & T30 \\
\hline Herida & T14.1 \\
\hline Úlcera venosa & $\mathrm{I} 83.0$ \\
\hline Úlcera arterial & $\mathrm{I} 33.9$ \\
\hline UEl no clasificada & $\mathrm{L} 97$ \\
\hline Úlcera en pie diabético & $\mathrm{L} 98.4$ \\
\hline UPP & L89 \\
\hline NANDA & Código \\
\hline Deterioro de la integridad tisular & $\mathrm{I} 1621 \mathrm{~A} \mathrm{(00046)}$ \\
\hline Deterioro de la integridad cutánea & $\mathrm{I} 1621(00044)$ \\
\hline
\end{tabular}

La variable fecha de cicatrización se obtuvo a través de la revisión de las notas de texto libre del seguimiento clínico, de la eCAP. Se decidió realizarla así porqué según nuestra experiencia, y la de Puig Girbau et al ${ }^{(9)}$, en ocasiones, los profesionales no cierran informáticamente los diagnósticos asociados a heridas, por lo que si éstos quedan abiertos existe riesgo de sobreestimar tanto la prevalencia como su duración.

Se revisaron también las notas del seguimiento clínico de las heridas diagnosticadas bajo taxonomía NANDA, para conocer su origen y así poder contabilizarlas, ya que a diferencia de los diagnósticos CIE éstos no aportaban información sobre la etiología de las heridas.

Para homogenizar la recogida de datos se elaboró una base de datos con Microsoft Excel 2007. Se realizó un análisis estadístico descriptivo de las variables con el 
programa IBM SPSS Statistics 22.0. Para los cálculos de prevalencia y tasa de incidencia se utilizó como denominador la población asignada a la ABS. Las variables fueron expresadas a través de frecuencias absolutas, frecuencias relativas, medianas, medias aritméticas y desviaciones estándares (DE) con intervalos de confianza (IC) del $95 \%$.

Para preservar la confidencialidad de los pacientes, en la base de datos se utilizó un código interno que permitiera identificar a cada paciente, garantizando su anonimato. Asimismo, la explotación no requirió ninguna actuación directa sobre los usuarios ni ninguna modificación en la eCAP. El proyecto fue aprobado por al comité de ética del Instituto de Investigación i Atención Primaria (IDIAP) de la fundación Jordi Gol y Gorina, vinculado al ICS.

\section{RESULTADOS}

Fueron localizados 416 registros de lesiones, de los cuales 43 (10,33\%) pertenecían a heridas crónicas. Un $53,48 \%$ eran mujeres. La edad media, de los pacientes con heridas crónicas, fue de 72,21 años (DE 5,5) (tabla II).

\begin{tabular}{|c|c|c|c|c|c|c|}
\hline \multirow{2}{*}{$\begin{array}{l}\text { Tipo de } \\
\text { lesión }\end{array}$} & \multicolumn{4}{|c|}{ Edad (años) } & \multicolumn{2}{|c|}{ Sexo } \\
\hline & $\begin{array}{l}>40 \\
\mathrm{n}(\%) \\
\end{array}$ & $\begin{array}{l}40-65 \\
\mathrm{n}(\%)\end{array}$ & $\begin{array}{l}66-80 \\
\mathrm{n}(\%)\end{array}$ & $\begin{array}{c}<80 \\
n(\%)\end{array}$ & $\begin{array}{c}\text { Hombres } \\
\text { n (\%) }\end{array}$ & $\begin{array}{c}\text { Mujeres } \\
\text { n (\%) }\end{array}$ \\
\hline Quemadura & $1(33,33)$ & $0(0)$ & $0(0)$ & $0(0)$ & $0(0)$ & $1(4,34)$ \\
\hline Herida & $2(66,66)$ & $4(44,44)$ & $\begin{array}{c}3 \\
(23,07)\end{array}$ & $1(5,55)$ & 7 (35) & $\begin{array}{c}3 \\
(13,04)\end{array}$ \\
\hline $\begin{array}{l}\text { Úlcera } \\
\text { venosa }\end{array}$ & $0(0)$ & $3(33,33)$ & $\begin{array}{c}5 \\
(38,46)\end{array}$ & $5(27,77)$ & $5(25)$ & $\begin{array}{c}8 \\
(34,78)\end{array}$ \\
\hline Úlcera & $0(0)$ & $0(0)$ & $0(0)$ & $1(5,55)$ & $0(0)$ & $1(4,34)$ \\
\hline $\begin{array}{l}\text { UEI } \\
\text { no } \\
\text { clasificada }\end{array}$ & $0(0)$ & $0(0)$ & $1(7,69)$ & $8(44,44)$ & $3(15)$ & $\begin{array}{c}6 \\
(26,08)\end{array}$ \\
\hline $\begin{array}{l}\text { Úlcera en } \\
\text { pie diabético }\end{array}$ & $0(0)$ & $1(11,11)$ & $1(7,69)$ & $0(0)$ & $2(10)$ & $0(0)$ \\
\hline UPP & $0(0)$ & $1(11,11)$ & $\begin{array}{c}3 \\
(23,07)\end{array}$ & $3(16,66)$ & $3(15)$ & $\begin{array}{c}4 \\
(17,39)\end{array}$ \\
\hline Total & $3(100)$ & $9(100)$ & $13(100)$ & $18(100)$ & $20(100)$ & $23(100)$ \\
\hline
\end{tabular}

La mediana de duración fue de 152 días y la media de 311 días (DE 135,38). Las que más se prolongaron en el tiempo fueron las UPP, con una media de 626 días (DE $643,78)$ y las que menos las heridas de región no especificada del cuerpo con 105 días de media (DE 84,31) (Figura 1).

Se estimó una prevalencia del 0,28\% y una incidencia del $0,24 \%$, calculada sobre una población de mayores de 15 años de 15.589 personas. Las úlceras venosas $(0,08 \%)$ fueron las más prevalentes. Las heridas de región no especificada y las úlceras 
venosas fueron las más frecuente, ambas con idéntica incidencia (0,06\%) (figura 2). El diagnóstico de herida de región no especificada del cuerpo englobó lesiones que cicatrizaban por segunda intención con etiología diversa: quirúrgicas, traumáticas, oncológicas o dermatológicas.

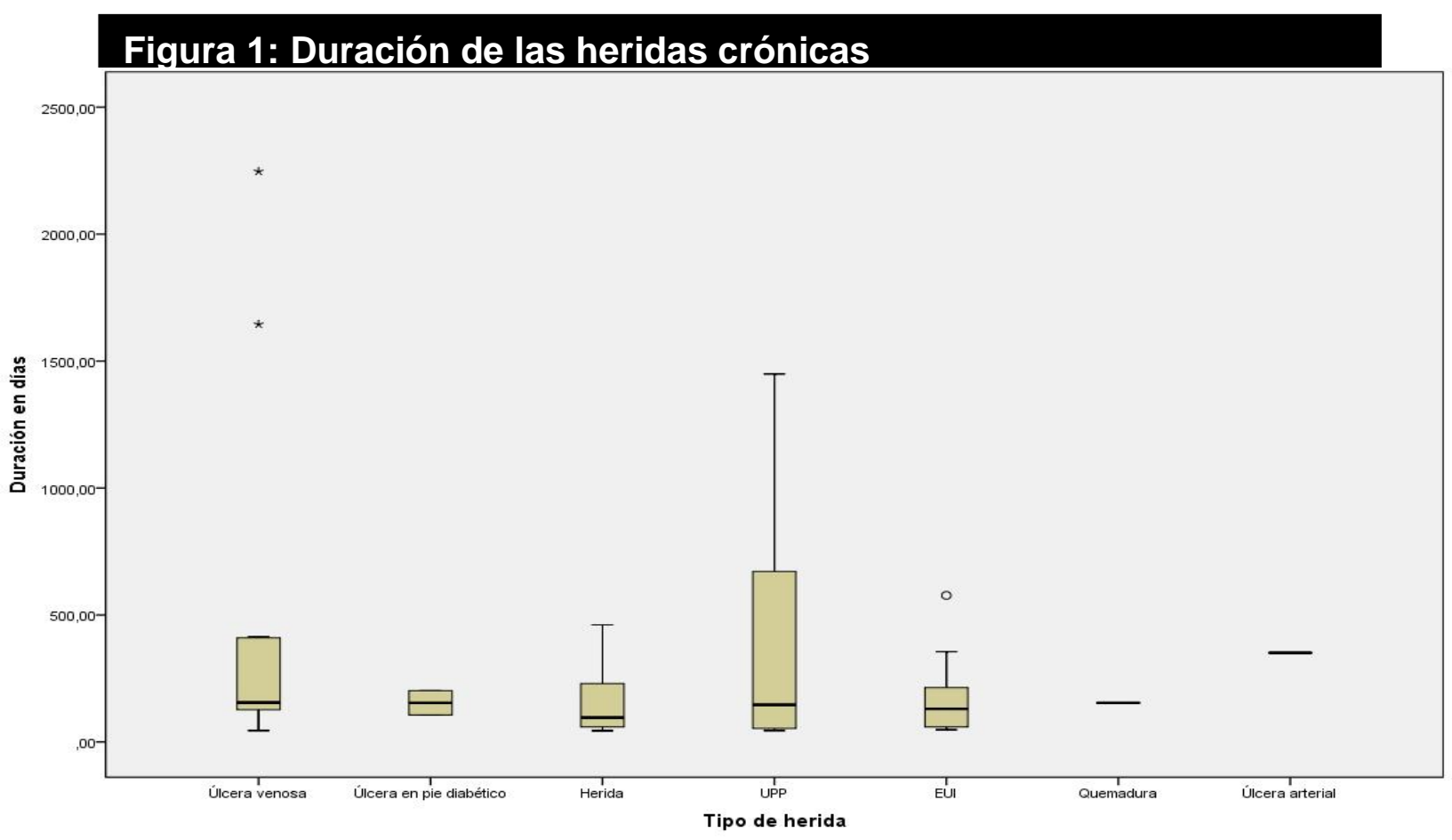

Un $30,23 \%$ de heridas crónicas pertenecían a pacientes atendidos en el programa de atención domiciliaria ATDOM. Se observó una diferencia estadísticamente significativa $(p<0,05)$ entre la media de duración de las heridas atendidas en el centro de salud y las atendidas a domicilio (tabla 3 ).

La etiología más prevalente en los pacientes de ATDOM fue la úlcera de extremidad inferior (UEI), con un $76,92 \%$. En la comparación de proporciones pudo observarse, que la proporción de pacientes atendidos en el domicilio que presentaban úlceras venosas, UEI y UPP, era significativamente superior a la proporción de pacientes atendidos en el centro de salud bajo el mismo diagnóstico $(p<0,05)$.

Todas las heridas tenían un solo diagnóstico ya fuera éste NANDA o CIE-10. Al realizar la revisión del seguimiento clínico se vio que no siempre coincidía el diagnóstico codificado, con el diagnóstico que se escribía en las notas de texto libre. Así, en el $25,58 \%$ de los registros se detectó que había una disonancia en el diagnóstico, es decir utilizaban un código $\mathrm{CIE}-10$ diferente al que luego mencionaban en el texto libre del seguimiento clínico. Bajo las etiquetas diagnósticas NANDA deterioro de la integridad cutánea y deterioro de la integridad tisular fueron diagnosticadas el $37,21 \%$ y el $4,65 \%$, de las heridas, respectivamente.

El seguimiento de todas las heridas incluidas en el estudio fue completo y no hubo pérdidas. 


\section{Figura 2: Prevalencia e incidencia de heridas crónicas}

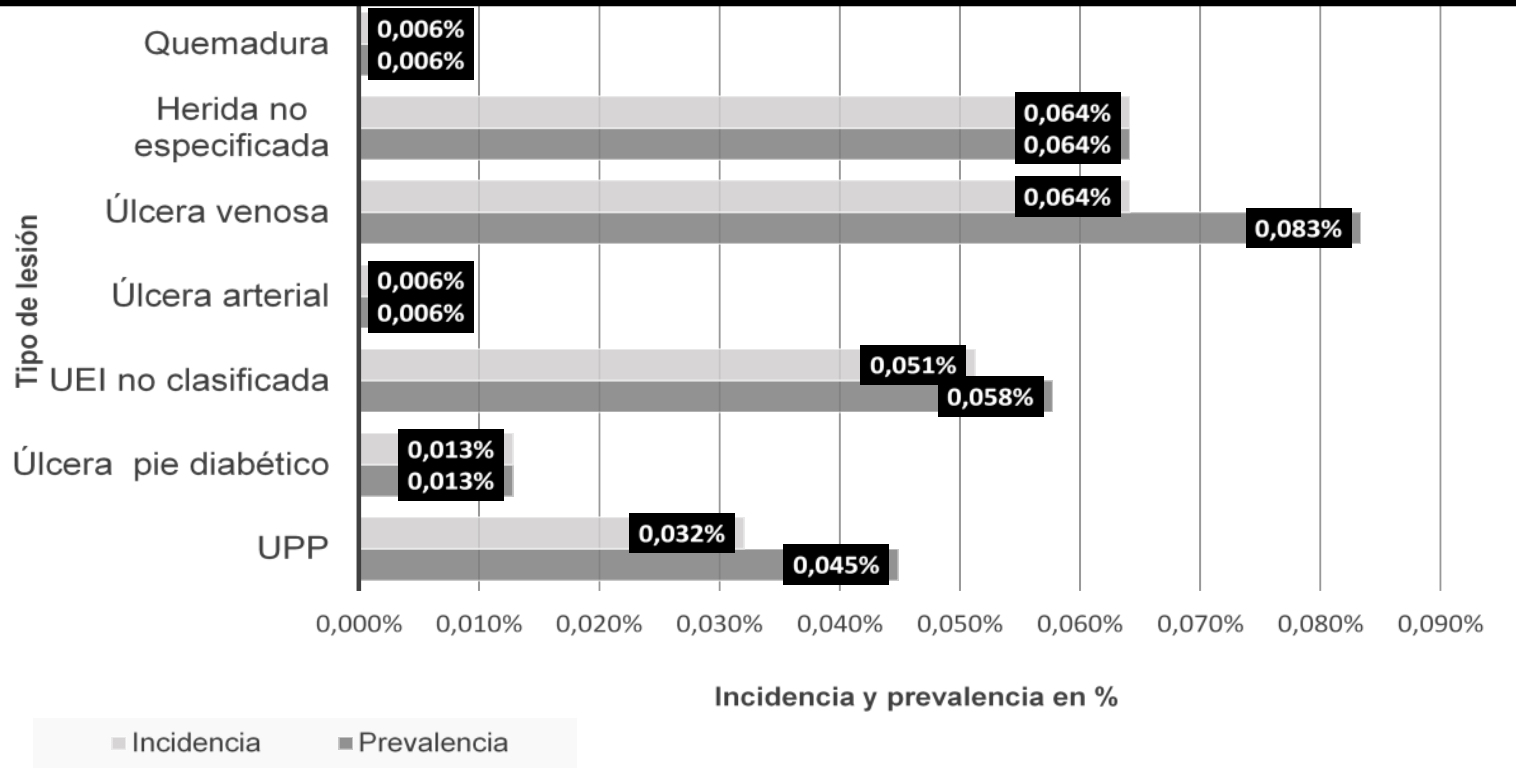

Tabla III: Distribución de las lesiones según lugar de atención de la cura y su duración

\begin{tabular}{|c|c|c|c|c|}
\hline \multirow[t]{2}{*}{ Tipo de lesión } & \multicolumn{4}{|c|}{ Lugar de atención de las curas } \\
\hline & \multicolumn{2}{|c|}{ CAP } & \multicolumn{2}{|c|}{ Domicilio } \\
\hline & $\mathrm{N}(\%)$ & $\begin{array}{c}\text { Duración media } \\
\text { en días (DE) }\end{array}$ & $\mathrm{N}(\%)$ & $\begin{array}{c}\text { Duración media } \\
\text { en días (DE) }\end{array}$ \\
\hline Quemadura & $1(3,33)$ & $59^{*}$ & $0(0)$ & $0(0)$ \\
\hline Herida & $10(33,33)$ & $105,10(84,31)$ & $0(0)$ & $0(0)$ \\
\hline Úlcera venosa & $8(26,67)$ & $232,5(137,30)$ & $5(38,46)$ & $401,4(267,09)$ \\
\hline Úlcera arterial & $1(3,33)$ & $196^{*}$ & $0(0)$ & $0(0)$ \\
\hline $\begin{array}{ll}\text { UEI } & \text { no } \\
\text { clasificada } & \end{array}$ & $4(13,33)$ & $195,75(152,67)$ & $5(38,46)$ & $490,4(571,11)$ \\
\hline $\begin{array}{l}\text { Úlcera en pie } \\
\text { diabético }\end{array}$ & $2(6,67)$ & $298,5(189,14)$ & $0(0)$ & $0(0)$ \\
\hline UPP & $4(13,33)$ & $138,5(102,44)$ & $3(23,08)$ & $\begin{array}{c}1276,33 \\
(1207,99)\end{array}$ \\
\hline Total & $30(100)$ & $173,39(56,87)$ & $13(100)$ & $637,54(383,10)$ \\
\hline
\end{tabular}




\section{DISCUSIÓN}

Evaluar la duración media de las heridas estudiadas resulta difícil por la amplia dispersión que existe en los datos, dispersión que ya muestran los diferentes estudios que engloban la revisión de Gonzalez-Consuegra y Verdú ${ }^{(10)}$ con duraciones que van desde los dos meses a los 50 años. Nuestros 311 días de media representan algo más que los 252 días que apunta un estudio realizado en Suecia ${ }^{(11)}$. Si analizamos sólo las UEI nos encontramos con una duración media de 315,8 días lo que estaría en consonancia con el estudio de Malaquias et al ${ }^{(12)}$ dónde apuntan que el $44,5 \%$ de las UEl estudiadas presentan una duración superior al año. Sin embargo, nuestros datos distarían mucho de los resultados del estudio de Edwards et al ${ }^{(13)}$ dónde sitúan la media en 12 semanas (84 días).

Por lo que respeta a las heridas atendidas en domicilio la duración media obtenida en el presente estudio supone casi seis meses menos que la obtenida en un estudio quebequés (14). Esta divergencia podría explicarse por las diferencias en la metodología del estudio, la etiología de las heridas, los productos utilizados en su tratamiento 0 las variables del entorno, ya sean éstas ambientales 0 socioeconómicas.

En nuestro estudio, prevalencia e incidencia son casi coincidentes, $0,28 \%$ y $0,24 \%$ respectivamente, algo que ya apuntan K. Kirketerp-Møller et al ${ }^{(8)}$. La prevalencia total se sitúa en un valor muy próximo al $0,29 \%$ que encontraron Ferrer-Sola et al ${ }^{(15)}$ en su estudio realizado en una comarca de la provincia de Barcelona, a pesar de las diferencias metodológicas entre ambos.

Sumando las prevalencias de úlceras venosas, úlceras arteriales y úlceras de extremidad inferior no clasificadas, obtenemos una prevalencia de UEI del $0,14 \%$ que coincide con los datos que ofrece La Conferencia Nacional de Consenso sobre las Úlceras de las Extremidad Inferior ${ }^{(16)}$. Mientras, nuestros datos de UPP, suponen menos de la mitad de lo que estima el último estudio nacional de la GNEAUPP (17). Esta notable diferencia podría deberse al infradiagnóstico de las UPP de grado I en nuestro equipo, aunque no podemos afirmarlo puesto que no se recogieron datos sobre los grados de las UPP.

Una limitación del estudio es el carácter retrospectivo, ya que podría generar una subestimación de la prevalencia e incidencia si, en el momento del diagnóstico, hubo heridas que se vincularon a códigos diagnósticos diferentes de los seleccionados. No contemplamos en la recogida de datos variables de tipo socioeconómico lo que nos podría haber ayudado a entender mejor los resultados. La explotación manual de datos supone una limitación ya que requiere más tiempo y presenta más riesgo de cometer errores.

La prevalencia de heridas crónicas es similar a la de otros estudios. Prevalencia e incidencia son casi coincidentes y destaca la prevalencia e incidencia de EUI por encima del resto de etiologías.

En conclusión, el tiempo de curación depende del tipo de herida y del lugar de atención. 


\section{REFERENCIAS}

1. Lazarus G, Valle MF, Malas M, Qazi U, Maruthur NM, Doggett D, et al. Chronic venous leg ulcer treatment: future research needs. Wound Rep Reg. 2014; 22(1):3442.

2. Situm M, Kolic M. Definicija i podjela atipičnih rana. Acta Med croatica. 2012; 66 Suppl 1:S5-11.

3. Formentini M, Fernandes LP. Factors that influence healing of chronic venous leg ulcers: a retrospective cohort. An Bras Dermatol. 2014; 89(3):414-422.

4. Cacicedo R, Castañeda C, Cossío F, Delgado A, Fernández B, Gómez MV, et al. Manual de Prevención y Cuidados Locales de Heridas Crónicas. Servicio Cántabro de Salud [Monografía en internet]. Cantabria: Servicio Cántabro de Salud; 2011 [acceso 21 Feb 2014]. Disponible en:

http://www.scsalud.es/documents/2162705/2163005/Manual+de+Preveci\%C3\%B3n+y +Cuidados+Locales+de+Heridas+Cr\%C3\%B3nicas_SCS.pdf

5. O'Meara S, Cullum N, Majid M, Sheldon T. Systematic reviews of wound care management: (3) antimicrobial agents for chronic wounds; (4) diabetic food ulceration. Health Technol Assess. 2000; 4(21):1-237.

6. Bateman S. Principles of preventative foot care. Br J Community Nurs. 2014; 19 Suppl 3: S30-38.

7. Kirketerp-Møller k, Zulkowski k, James G. Chronic Wound Colonization, Infection, and Biofilms. En Bjarnsholt T et al. Biofilm infections. Copenhagen: Springer; 2011. p. 11-24.

8. González-Consuegra V, Verdú Soriano J. Calidad de vida y cicatrización en pacientes con úlcera de etiología venosa. Validación del Charing Venous Ulcer Questionnaire, versión española (CCVUQ-e) y del Pressure Ulcer Scale for Healing, versión española (PUSH-e). resultados preliminares. Gerokomos. 2011; 22(3):131136.

9. Puig Girbau N, Teixidó Vargas C, Estébanez Perpina JL, Bosch Fortuna S. Prevalencia de lesiones en el ámbito de la atención primaria, a propósito de las variantes de registro. Metas Enferm. 2013; 16(2):6-10.

10. Gonzalez-Consuegra RV, Verdú J. Quality life in people with leg ulcers: an integrative review. J Adv Nurse. 2011; 67(5): 926-944.

11. Öien RF, Åkesson N. Bacterial cultures, rapid strep test, and antibiotic treatment in infected hard-to-heal ulcers in primary care. Scand J Prim Health Care. 2012; 30(4): 254-258

12. Malaquias GS Bachion MM, Sant'Ana SM, Dallarmi CC, Lino Junior RdeS, Ferreira PS. Pessoas com úlceras vasculogênicas em atendimento ambulatorial de enfermagem: estudo das variáveis clínicas e sociodemográficas. Rev Esc Enferm USP. 2012; 46(2): 302-310.

13. Edwards H, Finlayson K, Courtney M, Graves N, Gibb M \& Parker C. Health service pathways for patients with chronic leg ulcers: identifying effective pathways for facilitation of evidence based wound care. BMC Health Serv Res [serie en internet]. 2013 [acceso 21 Mar 2014]; 13 (86): [aprox 10 p.]. Disponible en: http://www.biomedcentral.com/1472-6963/13/86

14. Rodrigues I, Megié MF. Prevalence of chronic wounds in Quebec home care. Ostomy wound manag. 2006; 52(5):46-57.

15. Ferrer-Solà $M$, Chirveches-Pérez E, Molist-Señé $G$, Molas-Puigvila $M$, BesolíCodina $A$, Jaumira-Areñas $E$, et al. Prevalencia de las heridas crónicas en una comarca de la provincia de Barcelona. Enferm Clin. 2009; 19(1): 4-10. 
16. Conferencia Nacional de Consenso sobre Úlceras de la extremidad inferior. Documento de consenso CONUEI. Barcelona: EdikaMed; 2009.

17. Pancorbo-Hidalgo PL, García-Fernández FP, Torra Bou JE, Verdú Soriano J, Soldevilla-Ágreda JJ. Epidemiología de las úlceras por presión en España en 2013: 4. ․ Estudio Nacional de Prevalencia. Gerokomos. 2014, 25(4):162-170.

Recibido: 15 de febrero 2016;

Aceptado: 15 de abril 2016

ISSN 1695-6141

(๖) COPYRIGHT Servicio de Publicaciones - Universidad de Murcia 